\title{
Performance Analysis of OFDM Based Cooperative Communication over Nakagami Fading Channel
}

\author{
S. R. Sabuj ${ }^{1}$, S.Das ${ }^{2}$ and Md. Jakir Hossain ${ }^{3}$ \\ ${ }^{l}$ Department of EEE, Bangladesh University, Dhaka, Bangladesh \\ ${ }^{2}$ Department of EEE \\ ${ }^{3}$ Department of CSE, Metropolitan University, Sylhet, Bangladesh \\ saifuriict@gmail.com,skshna@gmail.com andmd.jakir.sub@gmail.com
}

\begin{abstract}
Cooperative communication is a fourth generation novel scheme promising significant capacity and multiplexing gain in wireless communication. This paper derives the carrier to noise interference ratio (CNIR) and average bit error rate (BER) for orthogonal frequency division multiplexing (OFDM) based amplify and forward (AF) and decode and forward $(D F)$ scheme over Nakagami fading channels. Numerical resuln are provided to show the significant improvement of DF in OFDM based cooperative communication due to same frequency offset and phase noise.
\end{abstract}

Keywords: Amplify and forward, Decode and forward, Erequency offset, Orthogonal frequency division multiplexing and Nakagemifading

\section{Introduction}

Cooperative communication i a capable key for the high data-rate exposure and enhanced capability with nophysical antenna an ay in upcoming wireless communication scheme and it is likely to be a key enabling technology for efficient spectrum use in fourth generation (4G). $4 \mathrm{G}$ has wo major benefits: cooperative relay scheme and combating the impairments of the rireless channel in this technology. The proposed schemes can increase the system reliability by achieving cooperative relay schemes and also increase their effective quanty of service via cooperation.

Various relay sehemes haye been proposed to achieve the benefit such as amplify and forward (AF) decode and forward (DF), coded cooperation (CC) and Hybrid DF-AF relay scheme $[1,2]$. The advantage of cooperative relay scheme is effortlessness and low cost implementation Orthogonal frequency division multiplexing (OFDM) is a multiplexing technique and sensitive to normalized frequency offset and phase noise which obliterates the orthogonality among subcarriers and cause inter carrier interference (ICI). OFDM based on single relay cooperative transmission technique promises significant performance-gains in terms of link reliability, spectral efficiency, system capacity and transmission range. The Nakagami- $m$ fading channel is commonly used to model the multipath fading radio transmission in urban areas, where the random fluctuations of the instant received signal power are very common and fast [3]. The benefits of Nakagami- $m$ fading cover a wide range of multipath fading channels by varying its fading parameter $\mathrm{m}$. It has greater flexibility in matching some experimental data than the Rayleigh, Rician fading [4, 5].

In this paper, we present without and with diversity expressions for carrier to noise interference ratio (CNIR) of OFDM based DF and AF relaying protocol with single number of relays that uses maximum ratio combining (MRC) technique at the destination for both cases. The average bit error rate (BER) expression has been derived for the proposed system with M-QAM modulation scheme in Nakagami fading channel. In 
addition, we have also evaluated the effect of frequency offset and phase noise in our proposed model.

\section{System Model}

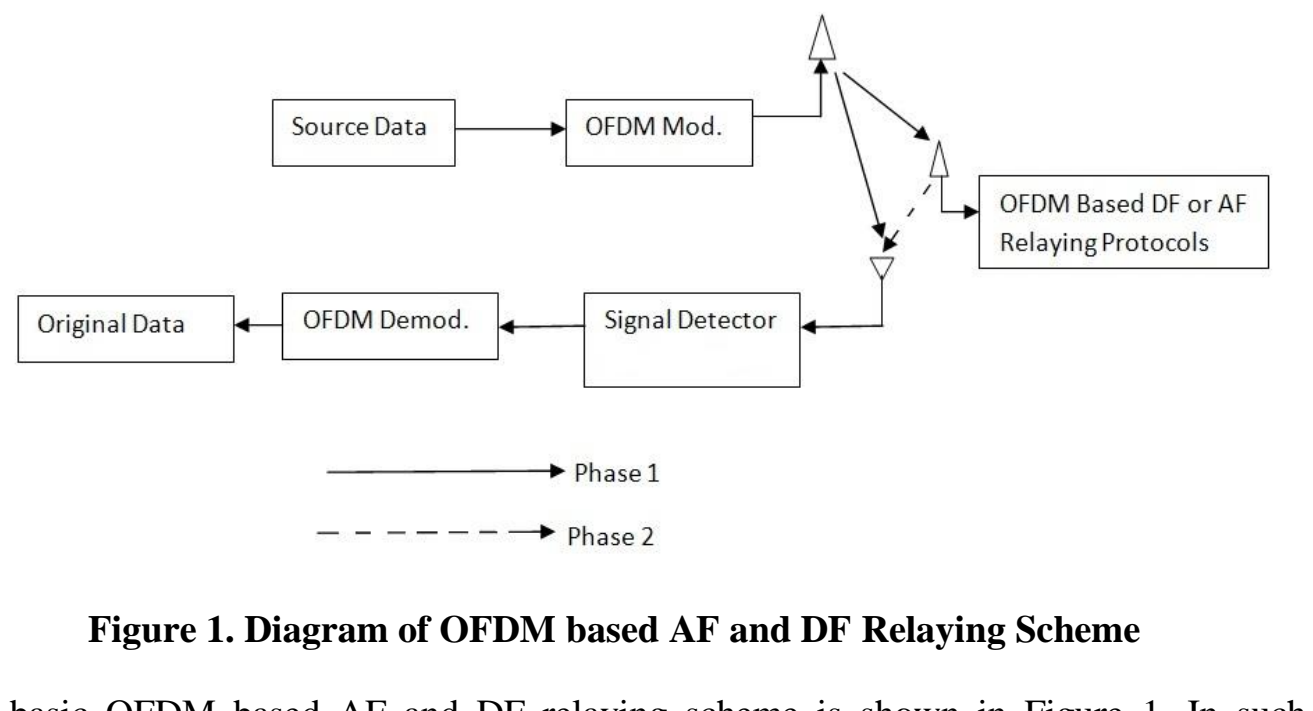

A basic OFDM based AF and DF relaying scheme is shown in Figure 1. In such OFDM based AF \& DF relaying technique the source datare modulated into OFDM modulation. Then it simultaneously tran mits information symbols. The outputs are subsequently sent into two ways in phase 1 and phase 2 . In phase 1 , the data transfer source to destination and source to relay directly on the other hand, in phase 2 data transfer from relay to destination. If DF scheme, relay receives the information symbols and decodes the original message. Then it fowards the message to the destination. In the $\mathrm{AF}$ method, relay receives the information and then amplifies the signal. Finally, it transmits the amplified rignal. One mobile acts as the source while the other mobile serves as the relay and the two mobiles may interchange their roles as source and relay anytime. In both cases, the relay enhances communication between the source and destination. At the reception side, eacb cooperative node has to forward its received signal through a yireless channel to the destination. In the destination section, the transmitted signals are detected with signal detection schemes and the detected signals are subsequently passed OFDM demodulator and decoded the original data.

\section{Theoretica Analysis of Single Relay}

\subsection{Decodd and Forward Relay Scheme}

In DF relaying scheme, the relay at first decode the received signal then forwards the received signal to the destination. In Phase 1, the source-relay and the source-destination received signals are given by [6]

$$
\begin{aligned}
& y_{k, s-r}=\sum_{l=0}^{N-1} x_{l, s} H_{l, s-r} Q_{l-k}+w_{r} \\
& =x_{k, s} H_{k, s-r} Q_{0}+I_{I C I}(s-r)+w_{r} \\
& y_{k, s-d}=\sum_{l=0}^{N-1} x_{l, s} H_{l, s-d} Q_{l-k}+w_{d}^{(1)} \\
& =x_{k, s} H_{k, s-d} Q_{0}+I_{I C I}(s-d)+w_{d}^{(1)}
\end{aligned}
$$


Where, ${ }_{I C I}(s-d)=\sum_{l=0, l \neq k}^{N-1} x_{l, s} H_{s-d, l} Q_{l-k}$ and $I_{I C I}(s-r)=\sum_{l=0, l \neq k}^{N-1} x_{l, s}{ }^{H}{ }_{s-r} Q_{l-k}$ are the inter carrier interference of the source to destination (s-d) and the source to relay (s-r). Here $x_{l}, H_{k, s-r}$ and $H_{k, s-d}$ are the transmitted information symbol, channel coefficients of the source to relay and the source to destination links respectively. The terms $w_{r} \sim \mathrm{CN}\left(0, \sigma_{r}^{2}\right)$ and $w_{d}^{(1)} \sim \mathrm{CN}\left(0, \sigma_{d}^{2}\right)$ is the AWGN at the relay and the destination of phase 1 respectively. $\sigma_{r}^{2}$ and $\sigma_{d}^{2}$ are the relay and destination noise variance. $\varepsilon$ is the normalized frequency offset and is given by $\Delta f T . T$ is the subcarrier symbol period.

$Q_{L}$ is defined as follows

$Q_{L}=\frac{1}{N} \sum_{n=0}^{N-1} e^{j\left[\left(\frac{2 \pi}{N}\right)(L+\varepsilon) n+\varphi(n)\right]}$

In Phase 2, the received signal at the destination can be expressed as

$$
\begin{aligned}
& y_{k, r-d}=\sum_{l=0}^{N-1} x_{l, r} H_{l, r-d} Q_{l-k}+w_{d}^{(2)} \\
& =x_{k, r} H_{k, r-d} Q_{0}+I_{I C l}(r-d)+w_{d}^{(2)}
\end{aligned}
$$

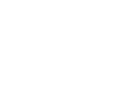

Where $I_{I C I}(r-d)=\sum_{l=0 l \neq k}^{N-1}{ }_{l}{ }_{r}{ }_{l}, C^{Q}{ }_{l-k}$ is the inter carrier inference of relay to destination, $|x|^{2}$ is the relay transmission power, $H_{k, r-d}$ is the channel coefficient of the relay to destination and $w_{d}^{(2)}$ is the AWGN at the destination of phase 2. The signals arriving at the destination can be combined by using with or without diversity technique. The carrier to noise interference ratio (CNIR) can be computed as

\subsubsection{Condition 1:Without Diversity}

In the condition of without diversity, the destination receives only the signal from relay. Using equation (4), CNIR can be written as,

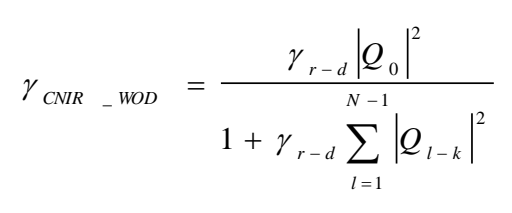

In the DF method, relay receives the source signal. Then it decodes the original message. So, we consider that $\left|x_{k, r}\right|^{2}=\left|x_{k, s}\right|^{2}$ and $\gamma_{r-d}=\frac{\left.\left|x_{k, s}\right|^{2}||_{k, r-d}\right|^{2}}{\sigma_{d}^{2}}$ is the carrier to noise ratio (CNR) from relay to destination. In the statistical properties [7], assuming average channel gain

$$
E\left[\left|H_{k, s-d}\right|^{2}\right]=E\left[\left|H_{k, s-r}\right|^{2}\right]=E\left[\left|H_{k, r-k}\right|^{2}\right]=1 \quad \text { and } E\left[\left|x_{k, r}\right|^{2}\right]=|x|^{2}
$$




\subsubsection{Condition 2: With Diversity}

In with diversity combining, the signals received in Phases 1 and 2, i.e., (2) and (4), can be optimally combined at the destination with maximum ratio combining (MRC) to obtain the output signal is expressed as

$y_{k, d, d o-\infty e}=\frac{H_{k, s-d}^{*} Q_{0}}{P_{l C C}(s-d)+\sigma_{d}^{2}} y_{k, s-d}+\frac{H_{k, r-d}^{*} Q_{0}}{P_{I C C}(r-d)+\sigma_{d}^{2}} y_{k, r-d}$

The effective average CNIR at the output of the OFDM based DF in MRC is given by $\gamma_{\text {CNIR }-w D}=\frac{\gamma_{s-d}\left|Q_{0}\right|^{2}}{1+\gamma_{s-d} \sum_{l=1}^{N-1}\left|Q_{l-k}\right|^{2}}+\frac{\gamma_{r-d}\left|Q_{0}\right|^{2}}{1+\gamma_{r-d} \sum_{l=1}^{N-1}\left|Q_{l-k}\right|^{2}}$

Where, $\gamma_{s-d}=\frac{\left.\left|x_{k, s}\right|\right|^{2}\left|H_{k, s-d}\right|^{2}}{\sigma_{d}^{2}}$ and $\gamma_{r-d}=\frac{\left.\left.\left|x_{k, s}\right|^{2}\right|_{k, r-d}\right|^{2}}{\sigma_{d}^{2}}$ are the CNR from source to destination and relay to destination. For an OFDM based DF in Nakagami m-QAM fading Channel, the probability density function (pdf) of the instantaneous channel CNIR is given by [8]

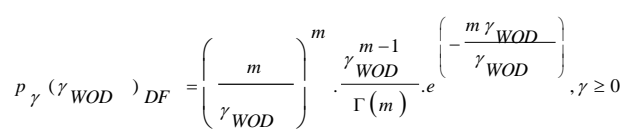

Where $\gamma=$ average CNIR . The bit error rae (BER) of Coherent M-QAM with twodimensional Gray coding over the AWGN hannel [9] $p_{b}\left(\gamma_{\text {WOD }}\right)_{D F} \cong 0.2 e^{\left(\frac{-3 \gamma_{\text {WOD }}}{2(M-1)}\right)}$

The average BER in OFDM based DF Nakagami- $m$ fading channel can be expressed as $p_{b}\left(\gamma_{\text {WOD }}\right)_{D F}=\int_{0}^{\infty} p_{b}\left(\gamma_{\text {WOD }} p_{\gamma}\left(\gamma_{\text {WOD }} d x\right.\right.$

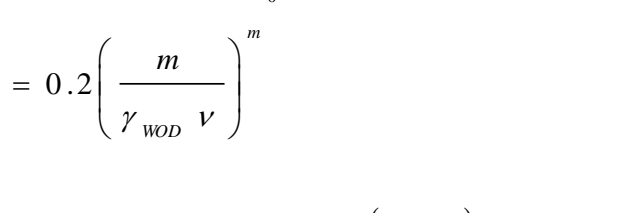

Where $v=\frac{3 \gamma_{W O D}+2 m(M-1)}{2(M-T)}$. The average BER expression is modified for the OFDM based DF with diversity over Nakagami- $m$ fading channel. CNIR is used in eq. (8).

\subsection{Amplify and Forward Relay Scheme}

In A relaying scheme, the relay receives a signal from source then forwards the receiyed signal to the destination in spite of the source-relay link quality. In Phase 1, the source-relay and the source-destination received signals are given by

$$
\begin{aligned}
& y_{k, s-r}=\sum_{l=0}^{N-1} x_{l, s} H_{l, s-r} Q_{l-k}+w_{r} \\
& =x_{k, s} H_{k, s-r} Q_{0}+I_{I C I}(s-r)+w_{r} \\
& y_{k, s-d}=\sum_{l=0}^{N-1} x_{l, s} H_{l, s-d} Q_{l-k}+w_{d}^{(1)} \\
& =x_{k, s} H_{k, s-d} Q_{0}+I_{I C I}(s-d)+w_{d}^{(1)}
\end{aligned}
$$


In phase 2 , the signal received by the relay that is attenuated and it is needed to be amplified before transmission another time. Assuming that the channel characteristic is estimated perfectly, the gain for the amplification can be calculated as follows. The desired received signal power is given by

$E\left[\left|y_{k, r}\right|^{2}\right]=\left|x_{k, s}\right|^{2}\left|Q_{0}\right|^{2}+P_{I C I}(s-r)+\sigma_{r}^{2}$

If the channel gain $\left|H_{k, s-r}\right|^{2}$ is known at the relay, the relay can multiply the received signal $y_{k, s-r}$ by the gain [2],

$$
\beta=\frac{1}{\sqrt{\left|x_{k, s}\right|^{2}\left|H_{k, s-r}\right|^{2}\left|Q_{0}\right|^{2}+P_{I C I}(s-r)+\sigma_{r}^{2}}}
$$

The relay signal is found after multiplication of gain that is expressed by, $x_{k, r}=\beta \times y_{k, s-r}$

The gain $\beta$ depends on the source-relay channel coefficient $H_{k, s-r}$. The relay forwards the signal $x_{k, r}$ to the destination, where the received signal can be expressed as

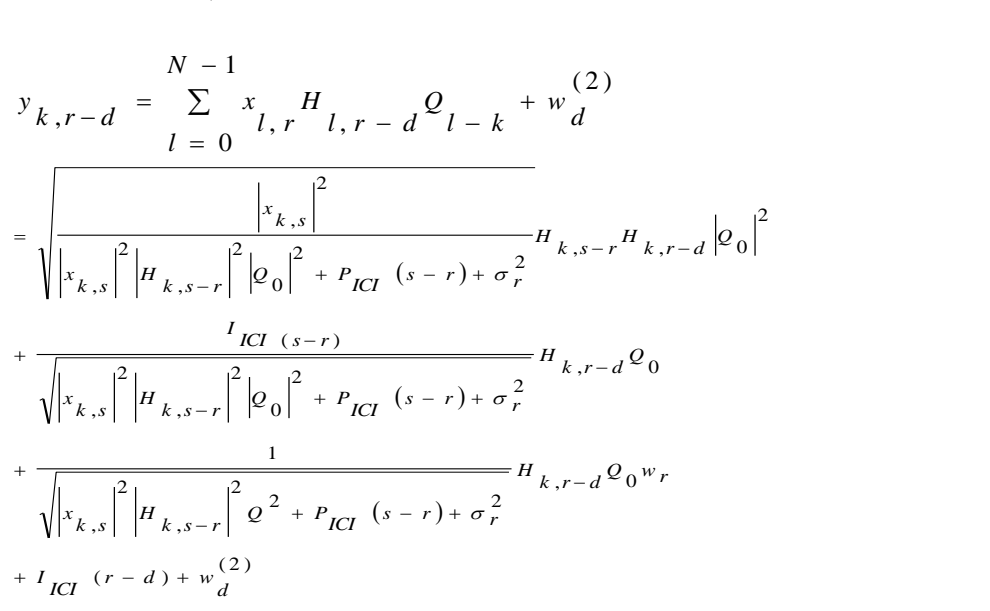

The signals arriving at the destination can be exploited for recognition with or without diversity combining.

\subsubsection{Condition 1:Without Diversity}

In the condition without diversity combining, only the signal $y_{k, r-d}$ will be utilized for detection at the destination. By (17), the received CNIR in this case can be computed as

$\gamma_{C N I R \_W O D}=\frac{\gamma_{s-r} \gamma_{r-d}\left|Q_{0}\right|^{4}}{\left.1+\gamma_{s-r}\left|Q_{0}\right|^{2}+\sum_{l=1}^{N-1}\left|Q_{l-k}\right|^{2}\right]+\gamma_{r-d}\left[\left|Q_{0}\right|^{2}+\sum_{l=1}^{N-1}\left|Q_{l-k}\right|^{2}\right]+\gamma_{s-r} \gamma_{r-d}\left[2 \sum_{l=1}^{N-1}\left|Q_{0}\right|^{2}\left|Q_{l-k}\right|^{2}+2 \sum_{l=1}^{N-1}\left|Q_{l-k}\right|^{2}\right]}$

\subsubsection{Condition 2: With Diversity}

In the condition with diversity combining, the signals received in (13) and (17), can be optimally combined at the destination employing maximum ratio combining (MRC) to obtain the output signal is expressed as, 


$$
\begin{aligned}
& y_{k, d}=\frac{H_{k, s-d}^{*} Q_{0}}{P_{I C I}(s-d)+\sigma_{d}^{2}} y_{k, s-d}
\end{aligned}
$$

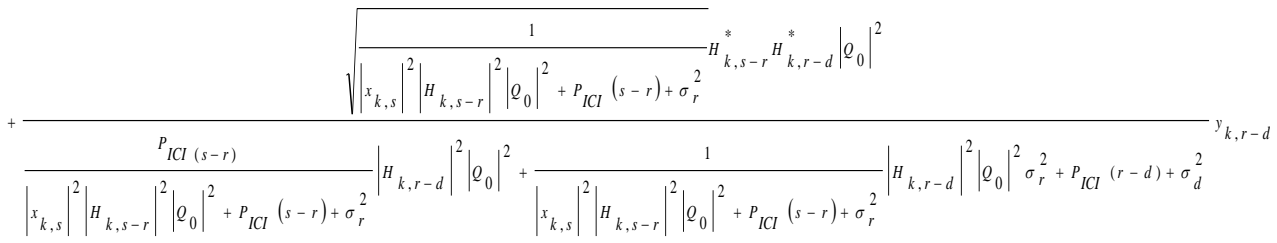

The effective CNIR of OFDM AF with diversity combining at the output of the MRC is given by

$$
\begin{aligned}
& \gamma_{C N I R-W D}=\frac{\gamma_{s-d}\left|Q_{0}\right|^{2}}{1+\gamma_{s-d} \sum_{l=1}^{N-1}\left|Q_{l-k}\right|^{2}} \\
& +\frac{\gamma_{s-r} \gamma_{r-d}\left|Q_{0}\right|^{2}}{1+\gamma_{s-r}\left|Q_{0}\right|^{2}+\sum_{l=1}^{N-1}\left|Q_{l-k}\right|^{2} 1+\gamma_{r-d}\left|Q_{0}\right|^{+} \sum_{l=1}^{N-1}\left|Q_{l-k}\right|^{2} 1+\gamma_{s-r} r_{r-d}\left|Q_{0}\right|_{l=1}^{N-1}\left|Q_{l-k}\right|^{2}+\left|Q_{0}\right|^{2} \sum_{l=1}^{N-1}\left|Q_{l-k}\right|^{2}+2 \sum_{l=1}^{N-1}\left|Q_{l-k}\right|^{2} 1}
\end{aligned}
$$

Whereas, $\gamma_{s-d}=\frac{\left|x_{k, s}\right|^{2}\left|H_{k, s-d}\right|^{2}}{\sigma_{d}^{2}}$ and $\gamma_{r-d}=\frac{\left.\left.\left|x_{k, s}\right|^{2}\right|_{k, r-d}\right|^{2}}{\sigma_{d}^{2}}$ are the CNR frôm sourrce to destination and relay to destination. The average BER in OFDM based AF Nakagami-m fading channel can be expressed as

$$
\begin{aligned}
& p_{b}\left(\gamma_{W O D}\right)_{A F}=\int_{0}^{\infty} p_{b}\left(\gamma_{W O D}\right) \times p_{\gamma}\left(\gamma_{W O D}\right) d \gamma \\
& =0.2\left(\frac{m}{\gamma_{W O D} v}\right)^{m} \\
& \text { Where, } v=\frac{3 \gamma_{W O D}+2 m(M}{2(M-1)} \text {. ThOD average BER expression is modified for the }
\end{aligned}
$$

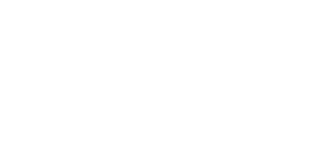
OFDM based AF with diversity over Nakagami-m fading channel. CNIR is used in eq. (20).

\section{Results \& Discussion}

In this section, we diseuss the simulation results of both without and with diversity technique in single relay cooperative communication. We focus on the CNIR and average BER performance analysis of both an AF \& DF relay schemes. The normalized frequency offset and phase noise is set to be 0.05 and 0.025 respectively. The M-ary, subcarrier, number of HFF and FFT are taken to be 4, 64, 64 and 64 respectively. 


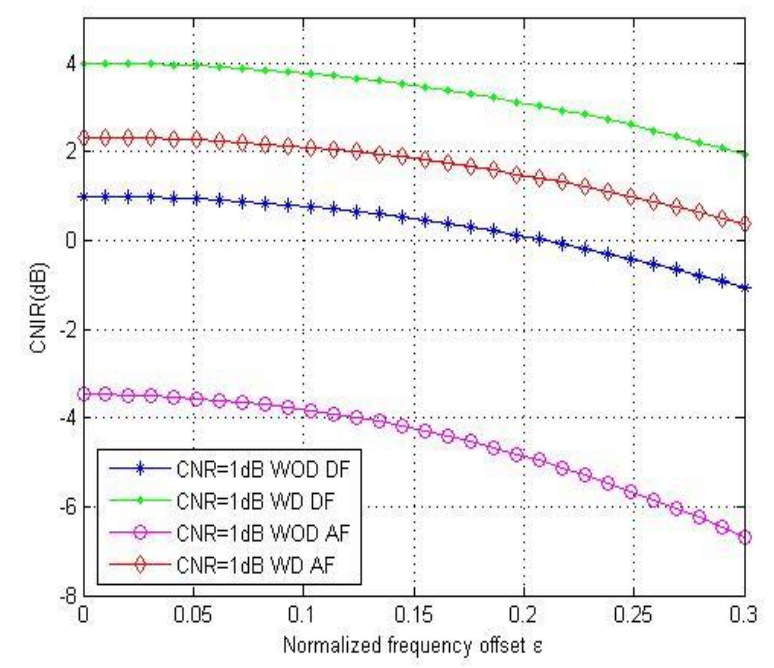

Figure 2. Plots of CNIR (dB) vs. Normalized Frequency Offset

The effect of normalized frequency offset is shown in Figure 2. Il also shows a performance analysis of the cooperative commun cation-system with implementation of $\mathrm{DF}$ and AF relaying protocol under without and with diversity technique. The system provides a quite satisfactory performance of $\mathrm{CNR}=1 \mathrm{~dB}$, WD DF relay scheme. For a typically assumed that normalized frequency ifset $\varepsilon$ value of 0.2 , the CNIR values are approximately $-4.808,0.1268,1.502$ and 3.137 in the ease of without and with diversity for $\mathrm{AF}$ and $\mathrm{DF}$ method. The performance o the system is improved in $\mathrm{CNR}=1 \mathrm{~dB} \mathrm{WD}$ DF by approximately $3.20 / \mathrm{dB}$ as compared to the $\mathrm{CNR}=1 \mathrm{~dB} \mathrm{WD} \mathrm{AF}$ respectively.

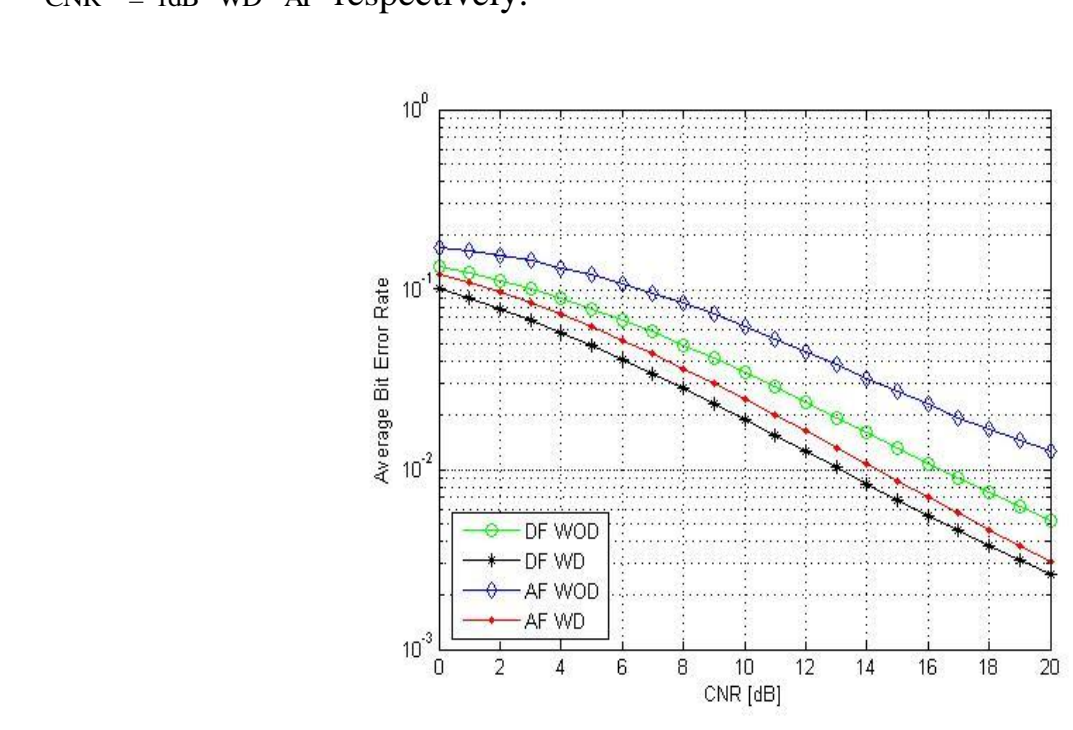

Figure 3. Average Bit Error Rate vs. CNR

Figure 3 shows the comparison between OFDM based cooperative DF and AF relay scheme for the Nakagami fading parameter, $m=1$. It is also noticed that the CNR value increases, performance has less average bit error rate to all schemes. The values of average bit error rate of $\mathrm{AF}$ WD, AF WOD, DF WD and DF WOD are approximately $0.03627,0.08369,0.02807$ and 0.04923 respectively at $\mathrm{CNR}=8 \mathrm{~dB}$. 


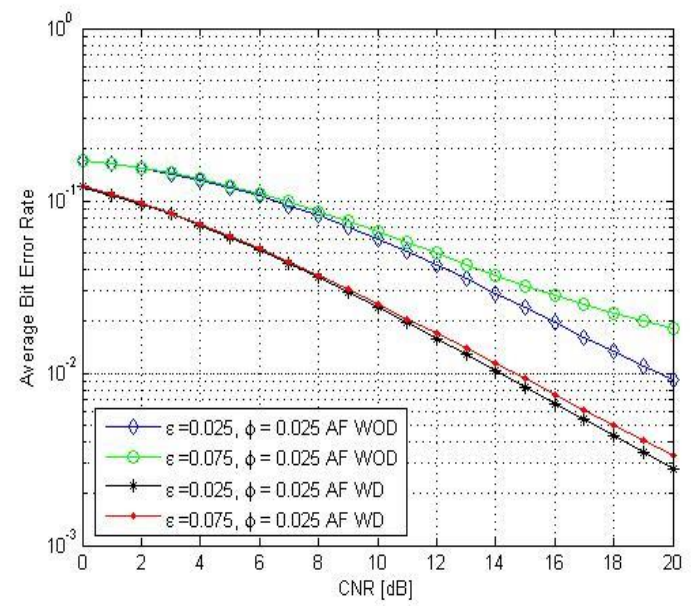

Figure 4. Effects of Frequency Offset on Average BER in AF Scheme

The effects of frequency offset on average bit error rate with differenl CNR are plotted in Figure 4. It is observed that AF relay scheme with diversity has better/performance than that of without diversity. Average BER decreases with increasing CNR and also for a particular CNR value, average BER increases as the frequency offset increases. For instance, at $\varepsilon=0.025 \mathrm{AF}$ WD, AF WOD and $\varepsilon=0.075 \mathrm{AF}$ WD AF WOD in OFDM based cooperative AF relay scheme value of average BER approximately are 0.01584, 0.04233 and $0.01693,0.04932$ when CNR value of $12 \mathrm{~dB}$. The scheme performance is increased in $\varepsilon=0.025$ AF WD by approximately 4.26 AB as compared to AF WOD.

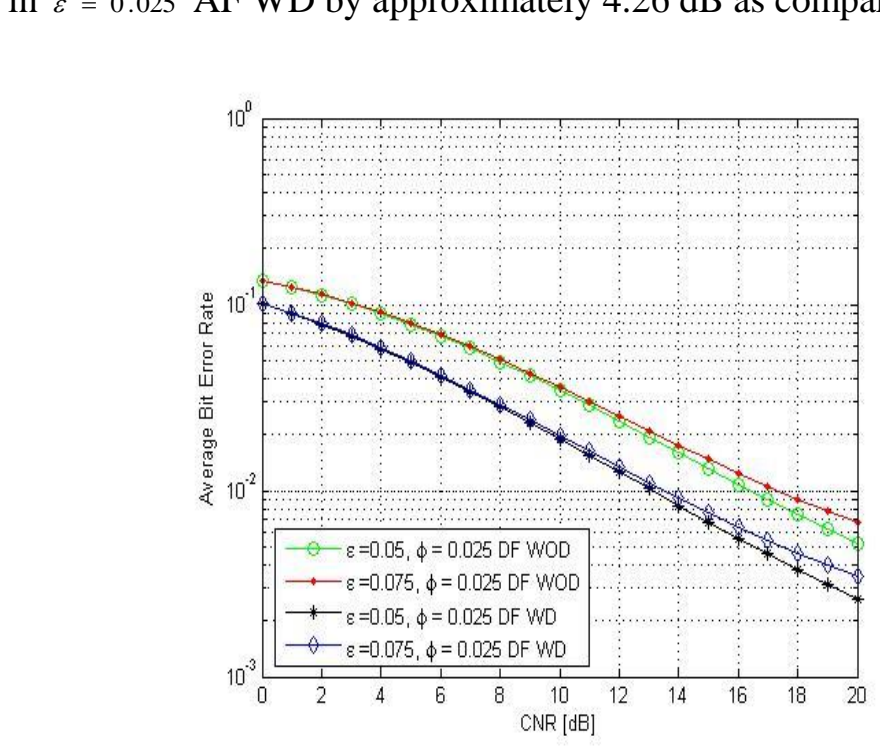

Figure 5. Effects of Frequency Offset on Average BER vs. CNR in DF Scheme

Figure 5 shows the performance analysis of average BER vs. CNR in DF relay scheme. DF with lower frequency offset is better than higher frequency offset. For a typically assumed CNR value of $10 \mathrm{~dB}$, the average BER values are approximately 0.02807, $0.04923,0.02893$ and 0.05055 in the case of $\varepsilon=0.05$ DF WD, DF WOD and $\varepsilon=0.075$ DF WD, DF WOD scheme. It is observable that at very high CNR, the various relay schemes performance is comparatively better under deployment of the OFDM based cooperative DF relay scheme. However, the best performance is that of the $\varepsilon=0.05, \phi=0.025$ DF WD relay scheme. 


\section{Conclusions}

The paper presents performance analysis for OFDM based cooperative communication without and with diversity technique over Nakagami fading channels. Some analytical expression for the CNR, CNIR and average BER of DF and AF scheme are obtained. Our numerical results show that the overall performance of the cooperative DF relay scheme in presence of frequency offset and phase noise is slightly better in contrast to the performance of AF relay scheme.

\section{References}

[1] H. Li, H. Nikookar and T. Xu, "OFDM Communications with Cooperative Relays", Communications and Networking Book, (2010).

[2] Y. W. Peter Hong, H. Wan-Jen and C. C. Jay Kuo, "Cooperative Communications and Networking", Technologies and System Design, Springer Science and Business Media, LLC, New York, ISBN-10: 1441971939, (2010).

[3] C. Chunxiao, Y. Wendong and C. Yueming, "Outage performance of OFDM-based selective decodeand forward cooperative networks over weibull fading channels", High Technology Letters, vol. 17, no. 3, (1995), pp. 285-289.

[4] N. C. Beaulieu and C. Cheng, "An efficient procedure for Nakagami-m fading simulation", IEEE Global Telecommunications Conference, vol. 6, (2001), pp. 3336-3342.

[5] J. Luo, J. Zeidler and S. McLaughlin, "Performance analysis of compact antenna arrays with MRC in correlated Nakagami fading channels", IEEE Trans. Veh Technol., vol. 50, no. 1(2001), pp. 267-277.

[6] Y.-S. Li, H.-G. Ryu, J.-W. Li, D.-Y. Sun, H.-Y. Liu, L. -J. Zhou and Y. Wat, "ICI compensation in MISO-OFDM system affected by frequency offset and phase noise", Wireless Commun., Networking and Mobile Computing ( WiCOM '08), vol. 5, no. 12, (2008), pp, $32-38$.

[7] V. K. Dwivedi and G. Singh, "An efficient BER analysis of OFDM systems with ICI conjugate cancellation method", PIERS Proceedings, Cambridge, USA, (2008).

[8] M. S. Alouini and A. J. Goldsmith, "Adaptive modulation ove Nakagami fading channels", Wireless Personal Communications: An International Journal, vol. 13 no. 1-2, (2000), pp. 119-143.

[9] T. Quazi and H. Xu "Performanceranalysis of adaptive M-QAM over a flat-flading Nakagami-m channel”, S Afr J Sci., (2011).

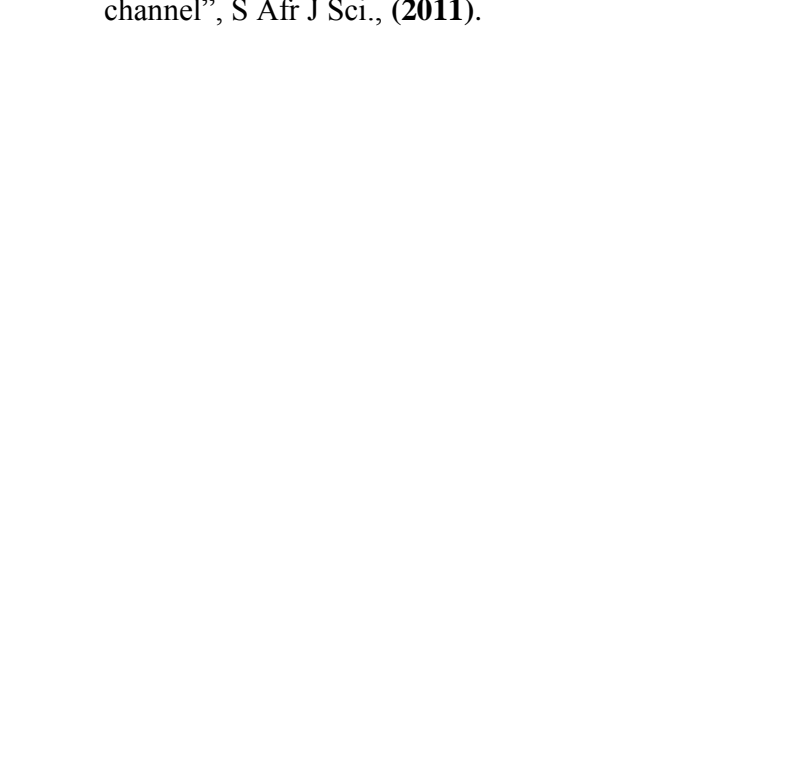


International Journal of Hybrid Information Technology

Vol.7, No.4 (2014)

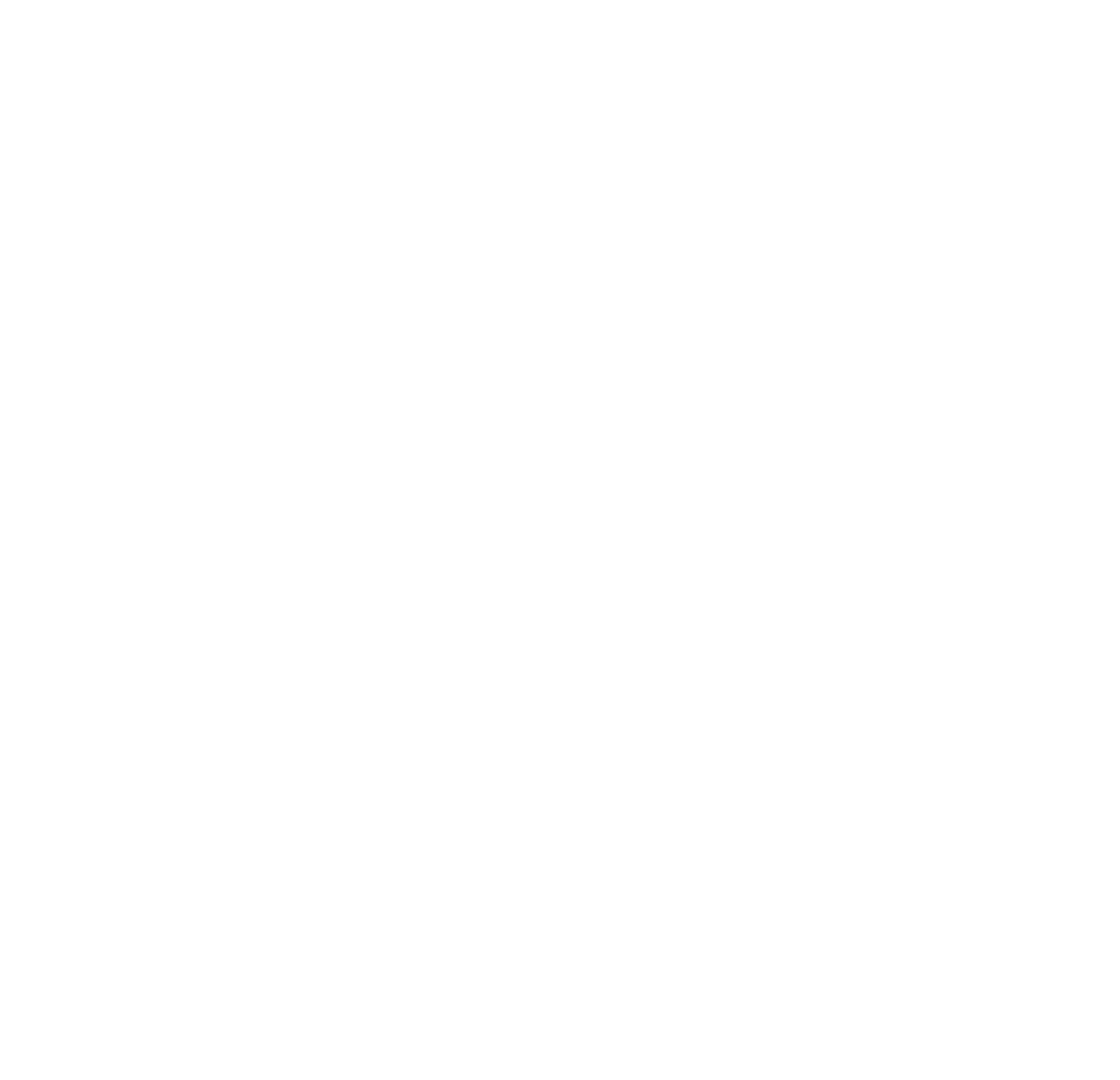

